\title{
Thema \\ La coordination entre registres sémiotiques : un enjeu important pour l'apprentissage des sciences
}

\author{
Alaric Kohler et Bernard Chabloz, HEP-BEJUNE
}

Cet article soulève un enjeu et argumente son importance dans les processus d'apprentissage des sciences : les élèves sont confronté.e.s à une diversité de registres sémiotiques (langue vernaculaire, mathématique, schémas, etc.) quìl leur incombe de coordonner. Cette tâche peut induire des situations de malentendu, comme nous tenterons de le montrer à l'aide de l'analyse a priori de quelques exemples issus des pratiques et manuels d'enseignement. Cette hypothèse est ensuite mise à l'épreuve d'une recherche collaborative, par l'intermédiaire de la conception d'un dispositif d'enseignement mi-fini centré sur l'introduction de pratiques de modélisation par les élèves au niveau secondaire II en Suisse.

Cet article ne présente qu'un extrait illustratif des résultats, en raison de la complexité des exemples et de la place limitée à ce type de publication. L'analyse présentée permet d'illustrer la pertinence de l'enjeu identifié lors des analyses a priori pour l'apprentissage de la physique, et invite à une utilisation plus délibérée et plus explicite de la coordination des registres sémiotiques dans la construction du milieu didactique lors de l'enseignement des sciences.

\section{Introduction}

Cet article soulève un enjeu et argumente son importance dans les processus d'apprentissage des sciences : les élèves sont confronté.e.s à une diversité de registres sémiotiques (langue vernaculaire, langage mathématique, schémas, etc.) qu'il leur incombe de coordonner. Il poursuit donc a un double objectif.

Premièrement, nous soulignons l'importance de détails spécifiques de la communication lors des pratiques d'enseignement dans une analyse sémiologique a priori de quelques exemples issus des pratiques et manuels d'enseignement. Ces exemples montrent que la tâche de coordination des registres sémiotiques comprend des difficultés intrinsèques qui peuvent induire des situations de malentendu entre élèves et enseignant.e.s.

Deuxièmement, nous illustrons la pertinence écologique des difficultés mises en évidence dans notre approche sémiologique, à partir de traces empiriques issues d'une recherche collaborative où les activités des élèves impliqué.e.s dans l'apprentissage de la physique font justement surgir un problème de coordination de registres sémiotiques différents lors de la représentation du même phénomène. L'exemple analysé illustre les difficultés dans la coordination de registres sémiotiques différents que rencontrent les élèves lors de la modélisation de la chute d'un objet.

En conclusion, nous proposons une utilisation plus délibérée et plus explicite de la coordination des registres sémiotiques dans la construction du milieu didactique, de manière à mobiliser les possibilités offertes par cette coordination comme une ressource préparée à l'attention des élèves. En effet, laisser dans l'implicite cet enjeu nous semble particulièrement propice à l'émergence de situations de malentendu (Kohler, 2015).

\section{Problématique}

La coordination des registres sémiotiques : un enjeu important

L'apprentissage des sciences est connu comme un défi, ce dont témoigne une abondante littérature de recherche se focalisant tantôt sur les conceptions des élèves (voir notamment Driver et al., 1985 ; Tiberghien, 2003), tantôt sur le changement conceptuel (voir notamment Carey, 1985, 2009 ; Vosniadou, 2013), ou encore sur les approches pédagogiques ou didactiques (voir notamment Bachelard, 1938/1972 ; Johsua et Dupin, 1993 ; Lemeignan et Weil-Barais, 1993 ; Driver et al., 1996 ; Astolfi et al., 1998 ; Greeno et Goldman, 1998).

Traditionnellement, les méthodes de recueil et d'analyse de données de la recherche en psychologie cognitive considèrent l'interprétation des tâches et des moyens sémiotiques distribués aux élèves non problématiques. 
Comme le remarque Weil-Barais (1993), lorsqu'un élève utilise le terme " force ", celui-ci est traité et analysé comme s'il avait le même sens pour l'expert.e physicien·e, l'enseignant·e, l'élève et la ou le chercheur.e. Par conséquent, la majorité de ces recherches ne considèrent pas dans le détail la médiation ${ }^{1}$ des pratiques d'apprentissage et d'enseignement étudiées par le langage et par les divers moyens sémiotiques utilisés. Sous-estimer l'importance de cette médiation conduit les chercheur.e.s à assimiler la pensée des élèves à la leur, en traitant le langage ou même la communication comme transparente, et constitue une tache aveugle de la recherche sur l'apprentissage (Perret-Clermont, 1992 ; Kohler, 2015). La psychologie sociogénétique a pourtant montré combien la construction des connaissances individuelles est intrinsèquement liée aux processus et milieux sociaux, et aux ressources culturelles qui y sont disponibles (voir notamment, Perret-Clermont et SchubauerLeoni, 1989 ; Gilly et al., 1999 ; Perret-Clermont et Carugati, 2004). La prise en compte des interactions dans l'apprentissage nécessite dès lors des chercheur.e.s une attention particulière aux processus de communication, et plus particulièrement, aux divergences d'interprétations des discours et des signes utilisés en milieu scolaire.

Quelques recherches s'intéressent en détail aux moyens sémiotiques utilisés en physique (voir notamment Halbwachs et Torunczyk, 1985 ; Brna et al., 2002) et permettent de montrer l'importance d'analyses détaillées du sens que les élèves et les enseignant.e.s donnent aux termes et aux autres moyens sémiotiques utilisés en classe de sciences. Ces recherches sont particulièrement importantes pour la théorisation de l'apprentissage des sciences : Amin (2009) montre par exemple combien le rôle du langage est sous-estimé dans le débat international sur le changement conceptuel, et comment cela a conduit les chercheur.e.s à une discussion sans issue.

Dans cet article, nous nous intéressons plus précisément aux liens que les élèves sont censé·e·s établir - et établissent effectivement - entre des moyens sémiotiques de registres différents (langue vernaculaire, langage mathématique, schémas, etc.) : cette coordination des registres sémiotiques a été identifiée par Duval (1995) comme une tâche cognitive essentielle au processus d'apprentissage en milieu scolaire. Néanmoins, les analyses détaillées de cette tâche de coordination sur des contenus particuliers sont encore très rares à notre connaissance.

\section{Faire pratiquer la modélisation par les élèves}

La modélisation a été identifiée comme une activité centrale de l'enseignement de la physique (voir notamment Martinand, 1992 ; Tiberghien, 1994) et des sciences en général. Gilbert (2013) distingue cinq approches faisant usage de modélisation dans l'enseignement, qui mettent en évidence l'implication relative des élèves : pour une majorité des approches, l'activité de modélisation est effectuée par les enseignant.e.s ou par les auteur·e.s des ressources sémiotiques (manuels, formulaires, etc.).

La recherche de Luc et Durey (1997) sur les usages de la modélisation montre, pour le cas particulier de travaux pratiques sur le haut-parleur, que ce sont rarement les élèves qui s'engagent cognitivement dans la modélisation : les modèles sont préparés pour elles et eux, et leur sont proposés sans qu'elles ou ils ne soient invité.e.s à fabriquer leur propre modèle. Introduire une pratique de la modélisation par les élèves a fait l'objet de plusieurs tentatives (voir notamment, Bécu-Robinault, 1997 ; Winther, 2006 ; Tytler et al., 2013) qui mettent en évidence le défi que constitue cet objectif.

C'est dans ce même objectif de stimuler des pratiques de modélisation par les élèves en milieu scolaire que nous avons conçu un dispositif d'enseignement mi-fini (Kohler et al., 2015) pour une recherche ${ }^{2}$ collaborative $^{2}$ centrée sur la question de l'appropriation du dispositif par les enseignant.e.s.

Nous ne présentons pas l'ensemble des résultats de cette recherche ici ni ne traitons la question de l'appropriation différente du dispositif par les enseignant.e.s, dans le but de discuter dans le détail l'une des hypothèses de l'analyse a priori effectuée pour la conception du dispositif : à savoir que les difficultés rencontrées lors de

1 Le concept de médiation est utilisé ici au sens de Vygotski (1934), repris par Bruner (voir notamment 1996) et une abondante littérature en psychologie culturelle qu'il serait trop long d'examiner ici. Cette littérature ne mobilise cependant pas le concept de médiation pour des analyses détaillées du sens que les élèves et les enseignant·e.s donnent aux moyens sémiotiques utilisés en classe de science comme nous le proposons ici : elle fournit des analyses à un niveau plus général, et se concentre le plus souvent sur l'importance d'une prise en compte d'une multitude de médiations culturelles, sociales et symboliques en général dans les processus psychologiques étudiés.

2 Projet de recherche institutionnel « Ingénierie didactique en physique, centrée sur la modélisation et la simulation : construction et évaluation d'un dispositif d'enseignement mi-fini (half-baked) pour le secondaire II ", Unité de Recherche 2, HEP-BEJUNE, http://modelisation-mi-fini.hep-bejune.ch. 
l'introduction d'activités de modélisation en classe consisteraient précisément à déléguer aux élèves la tâche de coordination de registres sémiotiques différents, et pour les élèves à réussir de telles coordinations. Cette hypothèse converge avec les résultats des travaux de Bécu-Robinault (2015).

\section{Méthodologie}

Cette investigation des enjeux de la coordination des registres sémiotiques est illustrative : elle vise à mettre en évidence l'importance de cette tâche cognitive dans l'apprentissage des sciences et la possibilité de situations de malentendu. Pour soulever un enjeu de la pratique et illustrer les possibles, cette démarche d'investigation ne répond pas à la logique, traditionnelle, qui s'oriente vers la mise en évidence de régularité et la généralisation des résultats (Astolfi, 1993).

La recherche a débuté par la conception d'un dispositif d'enseignement mi-fini sous la forme d'un site web ${ }^{3}$ comprenant des textes, des présentations audiovisuelles et des fichiers pour les programmes de modélisation (STELLA® et GEOGEBRA®). L'ensemble est orienté vers la délégation des activités de modélisation aux élèves.

La démarche de recherche repose sur l'ingénierie didactique (Artigue, 1988) qui distingue quatre phases pour les recherches portant sur les effets d'un dispositif d'enseignement :

1. Une analyse préalable des enjeux justifiant la conception d'un nouveau dispositif d'enseignement ;

2. Une analyse a priori des tâches et du fonctionnement escompté du dispositif ;

3. Une ou plusieurs expérimentations du dispositif ;

4. Une analyse a posteriori à partir des traces des activités des élèves et enseignant.e.s, permettant de confronter les hypothèses de l'analyse a priori à des évènements et processus documentés.

Ne pouvant présenter l'ensemble de ce long processus de recherche ici, nous nous focaliserons sur une seule hypothèse établie lors de l'analyse a priori - celle qui a trait à la coordination entre registres sémiotiques - dont nous présentons ci-dessous un exemple des réflexions permettant d'identifier l'enjeu exposé dans la problématique (un extrait de l'analyse préalable), quelques exemples d'analyse de schémas issus de manuels d'enseignement à disposition des écoles concernées par la recherche citée (un extrait de l'analyse a priori), et un bref extrait de la conversation enregistrée dans une classe (un extrait de l'analyse a posteriori).

Les données présentées à l'occasion de l'analyse a posteriori proviennent de l'expérimentation du dispositif d'enseignement mi-fini à laquelle ont participé deux enseignant.e.s et quatre classes (environ 80 élèves) de deux établissements du secondaire II de Suisse romande. Si les analyses présentées ci-dessous ne reprennent pas les mêmes contenus, c'est parce que nous avons choisi de présenter un extrait de chacune des phases de la recherche (voir ci-dessus), et que lors des analyses préalables et a priori nous n'avions pas encore connaissance des tâches proposées aux élèves par les enseignant.e.s qu'elles ou ils ont composées librement en s'inspirant du dispositif d'enseignement mi-fini. Nous tentons justement de montrer l'importance de la coordination des registres sémiotiques en tirant des liens entre nos premières analyses, effectuées avant la conception et la mise en œuvre du dispositif d'enseignement mi-fini, et l'illustration extraite de l'analyse a posteriori, qui reprend un évènement dans l'une des classes participant à l'expérimentation du dispositif.

Voici comment s'organisent ces résultats :

1. Premièrement, nous présentons l'analyse d'une anecdote révélatrice des enjeux de la recherche qui est un extrait de l'analyse préalable effectuée par les chercheur.e.s et qui a contribué à orienter la conception du dispositif sur la modélisation et la sémiotique ;

2. Deuxièmement, nous présentons une analyse d'extraits de manuels d'enseignement qui illustre les analyses a priori effectuées par les chercheur.e.s avant la recherche empirique. Ces analyses a priori ont permis de poser des hypothèses sur les difficultés auxquelles seraient confronté.e·s les enseignant.e.s mettant en œuvre le dispositif d'enseignement mi-fini proposé par les chercheur.e.s. Cette analyse permet également, dans ce texte, de montrer précisément le genre de problème auquel nous nous intéressons en thématisant les difficultés de coordination entre registres sémiotiques;

3. Troisièmement, nous présentons un extrait des résultats de la recherche empirique qui illustre une difficulté liée à la coordination des registres sémiotiques. Cet extrait comprend l'analyse d'une conversation enregistrée

3 Le dispositif d'enseignement mi-fini est disponible dans son intégralité à l'adresse suivante : http://modelisation-mi-fini. hep-bejune.ch. 
en classe de physique, qui montre l'émergence de ces difficultés dans une pratique scolaire effective visant à engager les élèves dans une activité cognitive de modélisation ;

4. Finalement, nous tentons de montrer les liens entre l'extrait produit à partir des données recueillies et l'hypothèse formulée lors de l'analyse a priori; comme il ne s'agit cependant que d'un bref extrait des résultats, nous ne prenons pas position sur l'importance relative de cette difficulté par rapport à d'autres difficultés. L'objectif de cette contribution consiste uniquement à mettre en évidence cette difficulté, à en fournir des exemples précis et concrets, de manière à donner les moyens de la repérer dans les pratiques et dans les données de recherche, et à illustrer sa pertinence écologique en milieu scolaire à l'aide d'un unique exemple.

Analyses

Analyse préalable : une anecdote révélatrice

Cet exemple relate une anecdote ${ }^{4}$ lors d'un examen oral de physique de première année de médecine. L'examinateur pose la question suivante :

«Pourquoi un caillou lâché du sommet de la tour Eiffel à Paris ne tombe-t-il pas à la verticale ?".

Le modèle théorique est celui de la chute libre, et la réponse escomptée est censée évoquer la rotation de la Terre et les forces de Coriolis. Face à quelques bredouillements de l'étudiante, visiblement en difficulté, l'examinateur lui fait esquisser rapidement au tableau noir un cercle pour représenter la Terre, indiquant les pôles, puis un triangle pour représenter la tour Eiffel. L'étudiante trace alors une ligne (verticale par rapport au tableau) pour représenter la trajectoire du caillou lâché du sommet de la tour Eiffel, reproduite en rouge sur la figure 1 (voir ci-dessous).

\section{Figure 1}

Reproduction de l'esquisse produite à l'occasion de l'examen oral cité.

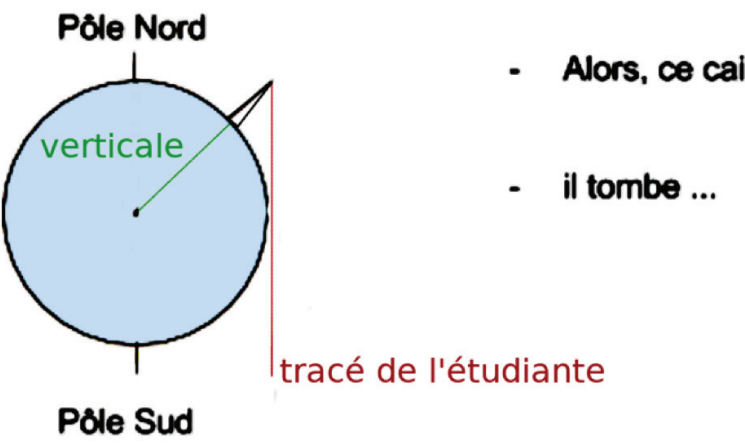

Pourquoi s'agit-il d'un problème de coordination de registres sémiotiques ? La question de l'examinateur est formulée en langue vernaculaire (ou registre discursif), et lorsqu'il propose de passer à un schéma au tableau noir, dans le but d'aider l'étudiante en offrant une autre médiation que celle de la langue orale, l'étudiante est confrontée au problème de la coordination entre les deux registres, puisqu'il s'agit désormais de répondre à la question de l'examinateur (registre discursif) sur le tableau noir à l'aide d'un schéma (registre graphique). Or, le registre graphique lui-même n'est pas utilisé de la même manière par l'examinateur, pour qui le centre de gravité du cercle qui représente la Terre indique la direction vers laquelle un objet chute à la surface de la planète (la verticale tracée en vert sur la figure 1) - autrement dit le "bas » - alors que pour l'étudiante c'est l'extrémité inférieure du tableau qui indique le "bas ", selon la convention souvent utilisée dans les illustrations qui place le sol sur la partie inférieure de la feuille de papier (la plus proche de soi), et le ciel en dans la partie supérieure (la plus éloignée de soi).

4 Jeannet, 1983, communication personnelle. 
Il y a cependant davantage en jeu dans cette anecdote qu'une référence à des conventions ou usages différents du dessin : les connaissances de physique devraient permettre à l'étudiante de prendre conscience que " tomber " correspond, sur le schéma représentant la Terre, à se rapprocher du centre de la planète. Si l'on demandait à l'étudiante pourquoi la verticale, pour l'objet qui tombe de la Tour Eiffel, est placée comme l'indique le trait vert sur la figure 1, il suffirait de répondre "par définition ». Autrement dit, la ligne verte constitue la traduction même du terme verticale (registre discursif) dans la représentation au tableau noir (registre graphique), mais pour pouvoir effectuer une telle opération de traduction, l'étudiante doit non seulement comprendre les deux registres et leurs conventions, mais également disposer d'un concept de verticale en physique qui permet justement de coordonner la langue dans laquelle s'expriment les savoirs et le monde, tel qu'il est perçu ou, dans notre exemple, lorsqu'il est représenté sous une forme schématique.

Cette anecdote montre - sans doute mieux qu'un long discours - le genre de défis que peut poser aux élèves la tâche de coordination de registres sémiotiques différents. Pour pouvoir accompagner les élèves dans ce processus, il nous semble indispensable d'analyser en détail, et pour chaque moyen sémiotique particulier, les difficultés que les élèves rencontrent ou peuvent rencontrer lors de l'interprétation des ressources qui leur sont soumises. Nous y contribuons ici par l'investigation de quelques exemples. Dans notre exemple ci-dessus, la difficulté est liée au fait que le tableau noir évoque une feuille de papier ${ }^{5}$ où, conventionnellement, le " bas » se trouve dans la partie inférieure alors que la verticale est définie en physique par le centre de gravité de la masse qui exerce une force sur l'objet et la position de l'objet.

\section{Analyse a priori de tâches de coordination}

À partir de schémas issus de manuels d'enseignement de la physique, nous avons procédé à une analyse de l'usage d'un moyen sémiotique particulier : la flèche. Cette étude montre que le rôle sémiotique de l'objet flèche n'est pas toujours le même, parfois même pour une seule illustration. Nous reprenons brièvement quelques résultats ci-dessous en faisant usage de la théorie du signe de Grize (1996) qui permet non seulement de distinguer la relation de signification entre le signifié et le signifiant, ${ }^{6}$ mais également le signifié conventionnel du sens spécifique que prend un signe dans une conversation ou un discours singulier. ${ }^{7}$ Ces sens varient également selon les interlocuteurs et interlocutrices, bien entendu, mais nous pouvons déjà proposer à l'occasion d'une analyse a priori une discussion générale de la diversité des sens que peuvent prendre des signes particuliers dans un contexte donné.

Commençons par un exemple issu du manuel Tavernier (Pierrard, 2009, p. 323) ${ }^{8}$, dont nous avons reproduit un schéma ci-dessous (figure 2). Ce schéma présente deux flèches dessinées de manière semblable, mais jouant deux rôles sémiotiques différents.

5 Nous pouvons noter que sur le tableau noir, la ligne rouge est bien verticale, alors qu'elle serait (probablement) horizontale sur une feuille de papier!

6 Le signifiant est la trace matérielle du signe, l'encre sur le papier ou la craie sur le tableau, alors que le signifié est la définition conventionnelle - si elle existe - de ce signifiant, tel qu'on la trouve dans le dictionnaire. Grize (1996) propose un modèle inspiré de Pierce qui ajoute à ces deux pôles un objet du signe, objet d'un discours particulier qui se distingue du sens conventionnel : c'est par exemple le sens que prend un mot pour un élève à un moment donné de son activité. La relation entre le signifiant et cet objet de discours est nommée dénotation. Pour certains signifiants, qui désignent quelque chose existant dans le monde, par exemple une tasse de café singulière, un quatrième pôle est ajouté au modèle : le référent. Le référent est alors la réalité désignée par le signifiant.

7 Ce problème nous paraît relever d'un enjeu générique aux malentendus dans l'éducation scientifique : pour un concept, le signifié est toujours abstrait (il s'agit souvent d'une définition), alors que les élèves ou étudiant-e.s le confondent souvent avec le référent, et pour cause : ils prennent le plus souvent connaissance du signifié à travers le référent, ne participant pas eux-mêmes à la longue élaboration historique du concept, processus au cours (et au terme) duquel le signifié en question hérite du nom habituellement attribué au référent. Ainsi, le langage utilisé précède souvent le concept lui-même. Ainsi, le langage quotidien (le rapport entre signifiant et signifié) interfere souvent avec le rapport entre signifiant et référent du langage scientifique, pour lequel le signifié differe de celui du langage quotidien : par exemple, une marguerite n'est pas une fleur (concept) pour le botaniste, mais « est » une fleur (notion) pour le non scientifique.

8 Il convient de préciser que tous les exemples fournis dans ce document sont présentés à titre d'illustration de propos théoriques émis par les auteurs à des fins de recherche et d'analyse. Ces exemples ne constituent donc ni une critique des ouvrages cités ni une évaluation prenant parti dans la compétition commerciale des manuels. Il convient d'insister sur le fait que les illustrations tirées des manuels cités ont été sélectionnées à dessein, et ne sont dès lors pas représentatives de la qualité des manuels dans leur ensemble. 
Figure 2

Exemple de "leviers simples ", extrait de Pierrard, 2009, p. 323.

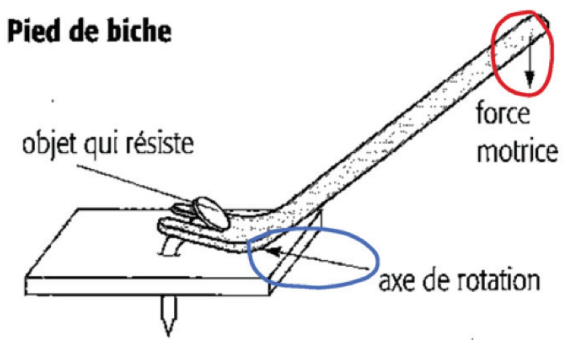

Une première flèche (entourée en rouge, à côté de "force motrice » sur la figure) désigne l'application d'une force et permet de faire figurer son sens, sa direction et peut-être son intensité - quoique d'une manière non graduée. Une seconde flèche (entourée en bleu, à côté de "axe de rotation ") désigne un endroit précis de l'esquisse. Deux rôles sémiotiques différents peuvent être identifiés à partir de ces deux usages de l'objet sémiotique flèche.

1. La flèche entourée de rouge remplit un rôle de modélisation : l'objet dessiné (la flèche) reprend certaines caractéristiques de l'objet modélisé (l'application d'une force), à savoir la direction, le sens, l'intensité (peutêtre) et le point d'application.

2. La flèche entourée de bleu remplit un rôle de pointage : elle se termine là où devrait se diriger l'attention de la lectrice ou du lecteur. En ce sens, elle constitue un déictique non verbal, au même titre que le mot ça ou ici, et a pour fonction de contribuer à construire une attention conjointe dans l'interaction sociale entre l'auteur.e du manuel et sa lectrice ou son lecteur.

Un autre phénomène intéressant est à relever sur l'esquisse ci-dessus : l'usage de deux objets sémiotiques différents pour le même rôle du pointage. La flèche est utilisée pour pointer un lieu de l'esquisse relié à la légende " axe de rotation ", et un trait simple est utilisé dans le même rôle de pointage, pour désigner la représentation visuelle du clou ou de l'" objet qui résiste".

Dans le schéma reproduit ci-dessus (cf. figure 2), nous avons vu que l'une des flèches jouait un rôle de modélisation de l'application d'une force. En tant que telle, cette flèche ne représente qu'un instantané de la dynamique du phénomène qu'elle modélise : un bref instant plus tard, elle aura peut-être une orientation ou une intensité différente. D'ailleurs, le propos de l'auteur·e n'est pas ici de suggérer une variation ou un mouvement, ce schéma modélisant une situation statique ou quasi statique. Pourtant, la ou le novice peut interpréter le schéma comme désignant une rotation du pied de biche, et la légende " axe de rotation " peut effectivement susciter ce malentendu. La convention qui, dans notre culture, associe un mouvement à l'objet flèche ne devrait donc pas être activée par la lectrice ou le lecteur. Si elle l'est, l'amalgame classique (Viennot, 1989) entre force et vitesse peut s'en trouver renforcé, d'autant plus que, précisément, il reste sensé dans les situations quasi statiques ou stationnaires, puisque force motrice et mouvement ont alors même direction et même sens. ${ }^{9}$

Dans un schéma modélisant une situation dynamique non stationnaire, on trouvera une même flèche pour représenter une force motrice qui, cette fois-ci, ne sera plus qu'exceptionnellement parallèle au mouvement : son caractère instantané devient alors essentiel à la compréhension de la description physique du phénomène. Ainsi, l'usage d'objets sémiotiques statiques comme une flèche - par contraste avec un document vidéo par exemple - conduit bien parfois à une modélisation du phénomène dynamique à un instant précis, mais cette réduction n'a pas la même portée selon le contexte. Or, il s'agit là souvent d'une décision - contrainte par les outils à disposition - qui reste implicite et peut également conduire les apprenant.e.s à des difficultés d'interprétation, notamment si elles ou ils s'attendent à ce que la flèche représente l'ensemble du phénomène dynamique plutôt qu'une partie de celui-ci (un instantané), et plus encore si rien ne les rend attentives ou attentifs à l'importance primordiale de cette instantanéité dans les situations non stationnaires.

9 Ces situations sont caractérisées par la quasi-absence d'accélération, et donc la quasi-disparition de l'inertie; c'est par exemple le cas du mouvement d'une bille dans la mélasse. 
À l'autre extrême de cet exemple peu congruent, les manuels contiennent également des exemples de pratiques qui différencient les signes en fonction du rôle que les auteur.e.s leur font jouer dans la communication. Par exemple, Benson (1999) utilise des flèches de formes et couleurs différentes pour représenter diverses grandeurs vectorielles (voir figure 3 ci-dessous).

\section{Figure 3}

Grandeurs vectorielles, extraits de Benson, 1999, p. 121 et 152.
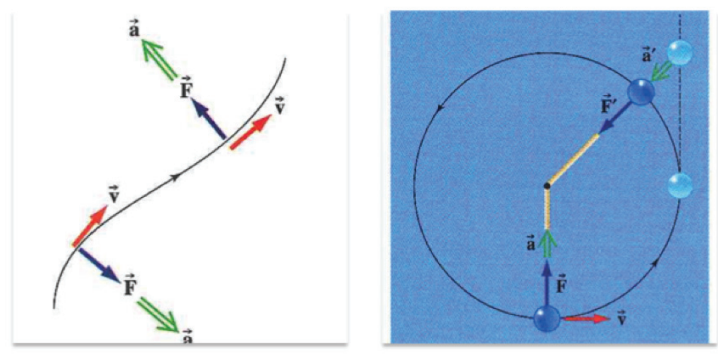

Cet artifice offre à l'enseignant.e la possibilité d'énoncer aux élèves le constat suivant pour les provoquer et s'enquérir de leur interprétation de ces objets sémiotiques :

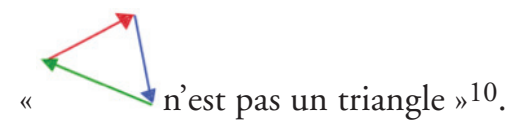

Même différenciation entre forces et vitesse dans la figure 4, avec toutefois une source potentielle de malentendus dans la mesure où les vecteurs obliques $\vec{k}_{i}$ et $\vec{R}_{2}$ ne sont pas de "nouvelles forces» subies par la voiture, mais bien les résultantes de forces horizontales (frottement et traction) et verticales (soutiens du sol) déjà présentes sur le schéma.

\section{Figure 4}

Forces subies par une voiture et vecteur vitesse, extrait de Martin, 1988, p. 43.

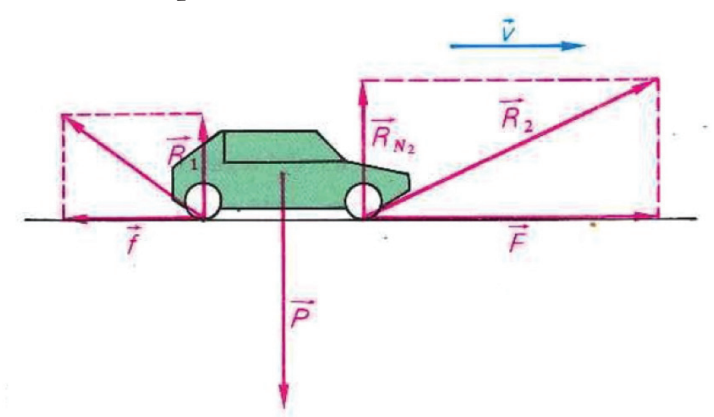

Les ambigüités relevées ci-dessus ne concernent pas seulement l'objet flèche. Le dessin d'un graphe, ${ }^{11}$ outil sémiotique couramment utilisé dans la pratique scientifique, peut également conduire à des difficultés d'interprétation semblables. Les exemples discutés ci-dessus nous permettent à présent d'aborder l'un de ces graphes que nous retrouverons justement dans l'analyse a posteriori - ce que nous ne savions pas au moment de produire l'analyse ci-dessous. La difficulté discutée ici a donc été anticipée au cours de la recherche.

10 En effet, d'abord un vecteur ne relie pas deux points. Mais surtout, chacune de ces trois flèches représente un vecteur sur la base de sa propre unité graphique (unité de force, respectivement d'accélération, respectivement de vitesse), si bien qu'aucun de ces vecteurs ne saurait être la somme des deux autres, même si le choix (implicite) des unités fait effectivement apparaitre un objet graphique qui pourrait modéliser un triangle (encore un concept, donc au signifié abstrait, et qui ne peut être "dessiné ».)

11 Dessin d'un graphe, ou graphique, nouvel exemple de concept : le dessin n'est pas le graphe. 
Prenons par exemple l'objet parabole - (reproduit en deux versions à la figure 5, ci-dessous) : avant que cet objet sémiotique ne devienne un outil pour les élèves, leur permettant d'exprimer une idée par le signe qu'il constitue, les élèves sont invité.e.s à lire ce signe complexe et à l'interpréter dans le sens escompté par leur enseignant.e. À cette occasion, plusieurs difficultés peuvent surgir.

\section{Figure 5}

Graphiques représentant chacun une parabole, alors que le premier (a) a pour abscisse et ordonnée des positions et le second (b) pour abscisse un instant et pour ordonnée une position.

$y[m]$$$
\text { a }
$$
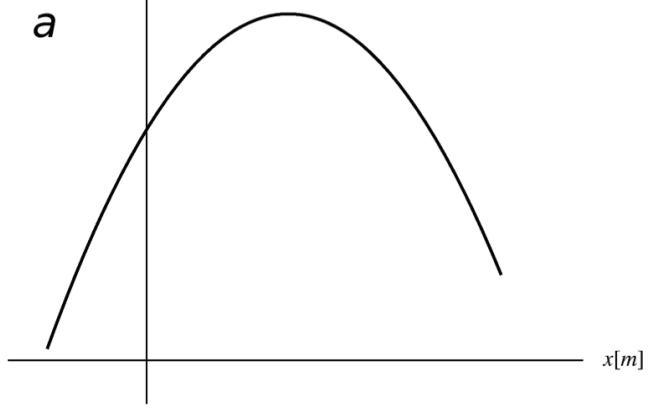

$x[m]$

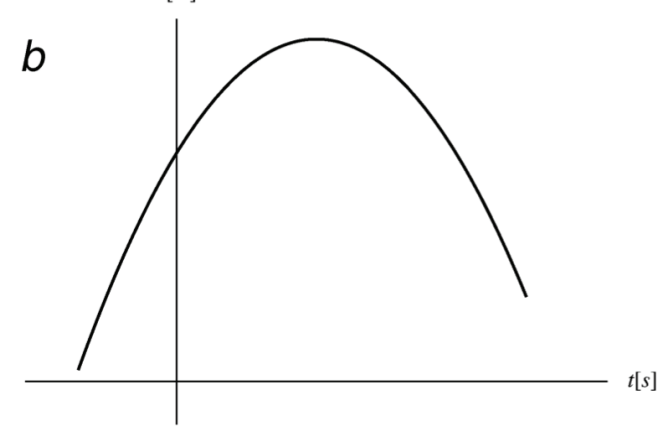

Prenons l'exemple de la figure 5 : les deux paraboles représentées ne désignent pas le même phénomène. La parabole de gauche (a) désigne la trajectoire d'un objet (lancé obliquement) en chute libre, alors que celle de droite (b) désigne l'horaire de la chute libre d'un objet lancé verticalement vers le haut.

Le risque d'ambigüité est patent : le dessin du graphe mobilisant immédiatement une représentation spatiale ou spatio-temporelle, le second graphique (b) sera interprété par les élèves comme le premier (a) qu'elles ou ils ont vu, soit comme une trajectoire. Deux remarques s'imposent.

1. La chute libre de la physicienne et du physicien est le mouvement d'un point matériel en l'absence de toute autre force que la pesanteur, ou dans un cas de figure où les autres forces se compensent exactement (ce qu'une simulation vise) : il ne s'agit donc pas d'un saut sans parachute ;

2. Un graphe n'est pas un dessin (même si le choix du mot graphe n'est sans doute pas innocent) : dessin $d u$ graphe (plutôt que graphique) est donc parfois écrit ici, à dessein, comme une nouvelle provocation à visée didactique.

Dans cet exemple, c'est la coordination d'un des deux graphiques présentés ci-dessus (Figure 5) avec le phénomène observé par les élèves qui leur permettrait de s’approprier le dessin de la parabole en tant qu'outil sémiotique. En outre, ces outils jouent chacun un rôle sémiotique spécifique et différent l'un de l'autre. En effet, l'objet parabole ne dénote en tant que tel ni une trajectoire, ni un horaire, de manière générale et conventionnelle : c'est un objet plus abstrait qui peut dénoter une relation entre deux grandeurs que l'utilisatrice ou l'utilisateur peut choisir selon ce qu'elle ou il a l'intention de communiquer. Pour s'approprier l'objet dessin de parabole en tant que signe permettant une variété d'usages, les élèves ne doivent pas seulement coordonner un graphique particulier avec le phénomène ou le concept correspondant, mais encore coordonner divers usages de paraboles dans divers graphiques.

Pour aller dans ce sens, la question de la coordination entre les deux graphes représentés à la figure 5 peut être posée aux élèves. Or, si la chute de l'objet est verticale, le premier graphe n'a pas lieu d'être. Et si l'objet a été lancé obliquement, alors une homogénéisation impliquerait que le second graphe présente la coordonnée y du premier en fonction du temps. Cette question peut contribuer à faire prendre conscience aux élèves que les paraboles sur les deux graphiques ont non seulement des référents différents, de telle sorte que les graphes ne sont pas les mêmes, mais également que tous les graphes ne sont pas forcément utiles pour tous les phénomènes : un graphe est une relation spécifique dont la pertinence est relative à un phénomène étudié. Cette prise de conscience peut contribuer à l'appropriation par les élèves de la parabole en tant qu'objet mathématique, ${ }^{12}$

12 Le processus escompté ici est une abstraction réfléchissante (Piaget, 1977) : le processus d'abstraction de la parabole du dessin du graphe singulier où elle est visuellement perçue par l'élève, au moment où il comprend qu'elle permet la description de relations singulières diverses dans des graphes différents, transforme par rétroaction la notion ou le concept de parabole de manière à en faire un outil descriptif de relations d'un certain genre. 
indépendamment de tout référent, et à leur faire comprendre le rôle instrumental d'un tel objet mathématique dans la modélisation du phénomène physique : le dessin de la parabole est ici utilisé comme un outil sémiotique pour représenter ou exprimer cette relation entre deux grandeurs. C'est l'auteur.e du modèle qui choisit le graphique en fonction de ce qu'elle ou il souhaite exprimer : cette dimension agentique de l'activité scientifique échappe très souvent aux élèves dans les pratiques institutionnelles d'enseignement, leur donnant alors la vague impression de ne rien y comprendre au moment où elles ou ils devraient (simplement) faire un choix et évaluer sa pertinence.

\section{Analyse a posteriori : illustration empirique de difficultés dans \\ La coordination}

L'extrait des traces enregistrées en classe et analysé ci-dessous provient d'une leçon centrée sur la modélisation, conçue par un enseignant participant à la recherche citée. Au moment de l'extrait, les élèves sont occupé·e.s à la construction d'un modèle désignant la chute libre d'un objet lancé. Une fois leur modèle construit, les élèves peuvent faire dessiner le graphique par l'ordinateur dans une fenêtre du logiciel STELLA®. Les élèves sont alors justement confronté.e.s au défi de lire ce graphique, qu'elles et ils ont elles-mêmes et eux-mêmes participé à élaborer : le lisent-elles et ils comme la représentation d'une trajectoire?

La transcription ${ }^{13}$ du Tableau 1 présente un discours de deux minutes précises, où l'enseignant improvise un discours de remédiation après avoir observé les difficultés que rencontrent ses élèves dans la tâche qui leur a été assignée. L'enseignant a notamment observé des élèves dessiner une courbe, sur leur graphique, qui reprend la forme de la trajectoire de l'objet alors que l'axe x représente le temps ; c'est comme si elles et ils confondaient les graphiques (a) et (b) de la figure 5 (voir ci-dessus).

\begin{tabular}{|c|c|}
\hline Locuteur.e & Registre verbal / REGISTRE GESTUEL \\
\hline \multirow{3}{*}{ Ens. } & $\begin{array}{l}\text { L'ENSEIGNANT LANCE SES CLEFS SUR UNE TABLE : ELLES DÉCRIVENT UNE PARABOLE, PUIS } \\
\text { GLISSENT À L'HORIZONTALE SUR LA TABLE }\end{array}$ \\
\hline & $\begin{array}{l}\text { de nouveau dans cet exemple-là . ce n'est pas du tout ça . pas du tout . je suis ironique mais c'est pas du tout ça. là } \\
\text { l'horizontale c'est l'axe du temps. donc il peut pas rouler sur le temps }\end{array}$ \\
\hline & L'ENSEIGNANT DÉPLACE SES CLEFS SUR LA TABLE À L'HORIZONTALE \\
\hline Ens. & $\begin{array}{l}\text { le temps s'écoule et l'objet a atteint le sol. je vais le dire pour tout le monde hein . mais la conclusion que vous avez } \\
\text { à peu près tous fait une fois c'est . et c'est normal de le faire. je crois que si on présentait cela à } 10 \text { classes dans les } \\
10 \text { classes il y aurait des gens qui feraient ces conclusions il y en aurait très peu qui ne les feraient pas . c'est qu'en } \\
\text { regardant le modèle. je vais juste le faire une fois ici pour }\end{array}$ \\
\hline \multirow[t]{2}{*}{ Ens. } & $\begin{array}{l}\text { L'ENSEIGNANT SE DÉPLACE EN DIRECTION DE L'ÉCRAN OÜ EST PROJETÉE LA FENÊTRE STELLA } \\
\text { on on voit ceci. }\end{array}$ \\
\hline & L'ENSEIGNANT POINTE UN GRAPHE AVEC UNE COURBE EN DEMI-PARABOLE \\
\hline \multirow[t]{2}{*}{ Ens. } & et on se dit ah. ah ouais effectivement c'est un objet . qu'on va lancer depuis une certaine hauteur \\
\hline & $\begin{array}{l}\text { L'ENSEIGNANT DÉSIGNE LE DÉBUT DU TRACÉ DE LA COURBE AU POINT O DE L'ABSCISSE } \\
\text { et on va le lancer comme ceci }\end{array}$ \\
\hline Ens. & $\begin{array}{l}\text { L'ENSEIGNANT LANCE SES CLEFS HORIZONTALEMENT QUI PARCOURENT UNE TRAJECTOIRE } \\
\text { SEMBLABLE AU TRACÉ DE LA COURBE }\end{array}$ \\
\hline \multirow[t]{2}{*}{ Ens. } & voilà puis ensuite \\
\hline & $\begin{array}{l}\text { L'ENSEIGNANT SE DÉPLACE DE L'AUTRE CÔTÉ DE L'ÉCRAN POUR FAIRE DISPARAITTRE LE MENU DU } \\
\text { PROJECTEUR QUI S'EST AJOUTÉ SUR L'IMAGE ET GÊNE LA VISIBILITÉ PUIS LES ÉLEVES ÉCLATENT } \\
\text { DE RIRE CAR LE MENU DU PROJECTEUR VIENT JUSTE DE DISPARAÎTRE AVANT QUE L'ENSEIGNANT } \\
\text { N'AIT PU INTERVENIR }\end{array}$ \\
\hline \multirow[t]{2}{*}{ Ens. } & miraculeux . on a lancé un objet comme ceci \\
\hline & $\begin{array}{l}\text { L'ENSEIGNANT TIENT LES CLEFS AU BOUT DES DOIGTS ET LEUR FAIT PARCOURIR UNE TRAJEC- } \\
\text { TOIRE SEMBLABLE A LA COURBE DU GRAPHIQUE TOUT EN LES TENANT, PUIS SUIT LAXE } \\
\text { DES ABSCISSES À PARTIR DU MOMENT OUं LA COURBE LE REJOINT, DANS UNE TRAJECTOIRE } \\
\text { HORIZONTALE - VOIR FIGURE } 6 .\end{array}$ \\
\hline
\end{tabular}

$\overline{13}$ Les points indiquent les pauses prosodiques, les «\# » un mot inaudible et le «? » une intonation montante. 


\begin{tabular}{|c|c|}
\hline Locuteur·e & Registre verbal / REGISTRE GESTUEL \\
\hline \multirow[t]{2}{*}{ Ens. } & $\begin{array}{l}\text { et puis ensuite il roule soi-disant comme une \#\#\# . il roule sur le sol et puis blebleble . non pas du tout. pas du tout } \\
\text {. ce n'est pas un objet que l'on lance comme ceci . c'est un objet qui est. lâché d'une certaine hauteur . ou lancé } \\
\text { vers le haut ou vers le bas mais pas du tout un lancé heu::: du point de vue horizontal }\end{array}$ \\
\hline & L'ENSEIGNANT FAIT UN GESTE DE PROPULSION DES CLEFS À L'HORIZONTALE SANS LES LÂCHER \\
\hline \multirow[t]{2}{*}{ Ens. } & c'est une chute verticale . un lancer comme ça \\
\hline & L'ENSEIGNANT LANCE LES CLEFS VERS LE HAUT, ELLES RETOMBENT DANS SA MAIN \\
\hline \multirow[t]{2}{*}{ Ens. } & ou un lâcher \\
\hline & L'ENSEIGNANT FAIT UN GESTE QUI ACCOMPAGNE LES CLEFS VERS LE BAS SANS LES LÂCHER \\
\hline \multirow[t]{2}{*}{ Ens. } & mais vertical . il n’y a qu’une seule dimension qui est influencée d'ailleurs ici \\
\hline & L'ENSEIGNANT DÉSIGNE SUR L'ÉCRAN L'AXE DES ORDONNÉES \\
\hline \multirow[t]{2}{*}{ Ens. } & $\begin{array}{l}\text { c'est la position en mètres. il n'y en a qu'une de position en mètres. et c'est la position verticale . c'est-à-dire l'altitude } \\
\text { si vous voulez quand vous le verrez . quand vous verrez la position initiale à dix . et ben l'objet est lâché ou lancé } \\
\text { d'une hauteur de dix . quand vous mettez vingt. ben il est lâché ou lancé d'une hauteur de vingt. d'accord ? ça }\end{array}$ \\
\hline & L'ENSEIGNANT DÉSIGNE LA COURBE DU GRAPHIQUE SUR L'ÉCRAN \\
\hline Ens. & $\begin{array}{l}\text { ça n'a rien à voir avec la trajectoire de l'objet. ce n'est pas la trajectoire . c'est le graphe de la position en fonction } \\
\text { du temps. c'est un modèle. la difficulté quand on lit des modèles c'est de ne pas se tromper entre le modèle et la } \\
\text { trajectoire . est-ce qu'on fait une petite pause? }\end{array}$ \\
\hline Élèves & non \\
\hline
\end{tabular}

Cet extrait montre que les élèves rencontrent justement les difficultés identifiées dans l'analyse a priori, consistant à confondre le registre graphique (produit par le logiciel) avec le comportement réel d'un objet observé en chute libre et décrivant une trajectoire en forme de parabole. Notons qu'une telle confusion est prévisible dès lors qu'on postule que dans un registre graphique l'interprétation figurative est plus immédiate que la construction d'une signification à partir d'un objet graphique abstrait : dans la figure 5, la parabole de gauche (a) " est " une trajectoire (représentation spatiale immédiate) alors que celle de droite (b) est un horaire (représentation spatiotemporelle non immédiate). Notons également que pour lever la confusion, l'élève doit lire ce qui est écrit sur les axes de coordonnées : le primat du langage graphique pourrait bien l'avoir dispensé.e de cette lecture. Cette confusion empêche la coordination des registres, que l'enseignant tente de soutenir par son discours en synchronisant justement ses gestes et la démonstration du comportement de l'objet. En effet, l'enseignant suit la courbe du graphique projeté sur l'écran avec les clefs qu'il a précédemment lancées pour montrer un exemple concret de trajectoire d'objet en chute libre.

\section{Figure 6}

L'enseignant suit la courbe du graphe projeté sur l'écran avec les clefs : la trajectoire de sa main est indiquée par un tracé pointillé en rouge, ajouté sur la photo par les auteurs.

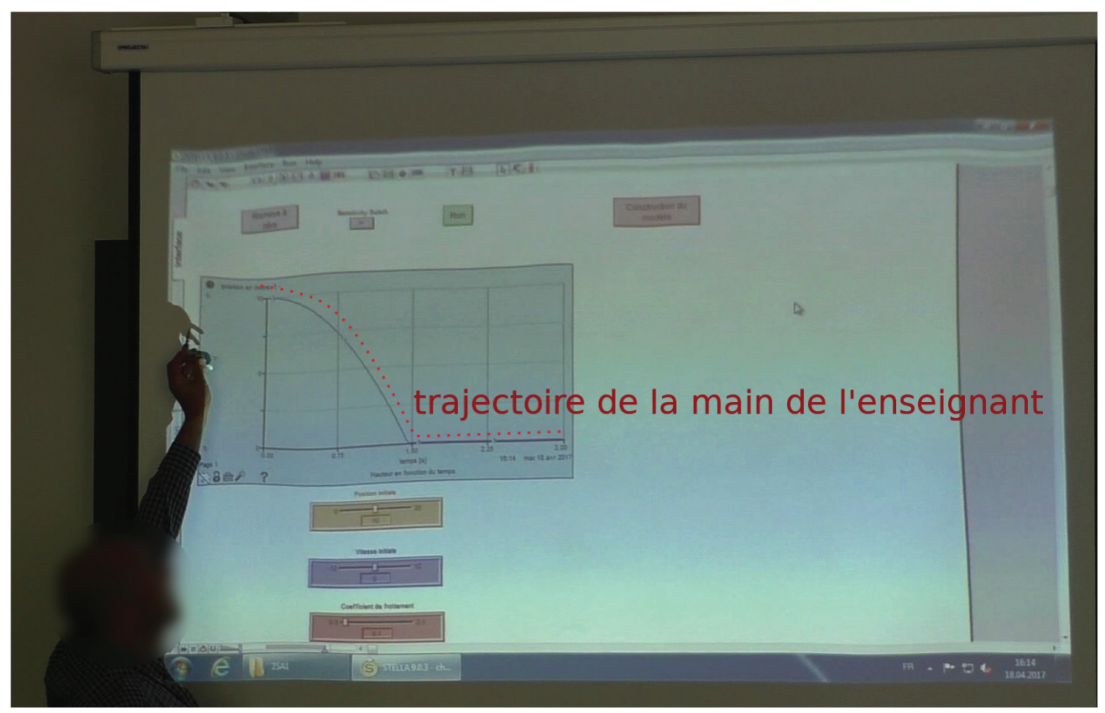


Nous ne disposons pas, dans cette recherche, des traces permettant de discuter l'efficience de cette coordination montrée aux élèves; par contre, le fait de voir l'enseignant insister sur cette coordination de manière spontanée lors de la leçon constitue déjà, de notre point de vue, un indicateur positif résultant de cette recherche collaborative, puisque les difficultés de coordination des registres sémiotiques différents sont rarement abordées explicitement dans les pratiques d'enseignement des sciences.

\section{Discussion}

Ces analyses illustratives ne permettent pas de généraliser les difficultés mises en évidence pour une population d'élèves, ni de présumer de l'importance du problème que pose la coordination des registres sémiotiques dans l'apprentissage des sciences relativement à d'autres sources de difficulté. Néanmoins, les quelques analyses $a$ priori que nous avons effectuées, et leur pertinence lors des analyses a posteriori que nous avons constatées en observant les élèves en situations scolaires naturelles dans une démarche de recherche collaborative montrent qu'il s'agit d'un enjeu tant au niveau théorique que pratique, et que de nombreuses recherches peuvent être conduites sur cette thématique. Le travail à faire est de grande ampleur, puisque ces analyses n'ont de sens que relativement à des moyens sémiotiques particuliers et en relation avec leurs spécificités. En outre, nous espérons avoir montré que les analyses a priori elles-mêmes, avant même l'analyse de traces empiriques, constituent un travail utile tant aux chercheur.e.s qu'aux enseignant.e.s, puisqu'elles permettent d'attirer l'attention sur d'éventuelles divergences d'interprétation qui, sinon, passent inaperçues, voire provoquent des difficultés d'apprentissage chez les élèves et des inférences erronées chez les enseignant.e.s et les chercheur.e.s.

\section{Références}

Amin, T.G. (2009). Conceptual Metaphor Meets Conceptual Change. Human Development, 52, 165-197.

Artigue, M. (1988). Ingénierie didactique. Recherches en Didactique des Mathématiques, 9, 281-308

Astolfi, J. (1993). Trois paradigmes pour les recherches en didactique. Revue Française de Pédagogie, 1, 5-18.

Astolfi, J.-P., Peterfalvi, B. et Vérin, A. (1998). Comment les enfants apprennent les sciences. Retz.

Bachelard, G. (1938/1972). La formation de l'esprit scientifique. Librairie Philosophique J. Vrin.

Benson, H. (1999). Physique 1. De Boeck Université.

Bécu-Robinault, K. (1997). Activités de modélisation des élèves en situation de travaux pratiques traditionnels: introduction expérimentale du concept de puissance. Didaskalia, 11, 7-37.

Bécu-Robinault, K. (2015). Un cadre épistémo-sémiotique pour concevoir des séances et analyser des pratiques d'étude et d'enseignement de la physique. Synthèse pour l'habilitation à diriger des recherches de l'Université de Toulouse. Université JeanJaurès.

Brna, P., Baker, M., Stenning, K. et Tiberghien, A. (2002). The role of communication in learning to model. Lawrence Erlbaum Associates.

Bruner, J. S. (1996). L'éducation, entrée dans la culture. Retz.

Carey, S. (1985). Conceptual Change in Childhood. MIT Press.

Carey, S. (2009). The Origin of Concepts. Oxford University Press.

Coquidé, M. et Le Maréchal, J.-F. (2006). Modélisation et simulation dans l'enseignement scientifique : usages et impacts. Aster, 43, 7-16

Driver, R., Guesne, E. et Tiberghien, A. (1985). Children's ideas in science. Open University Press, Milton Keynes.

Driver, R., Leach, J., Millar, R. et Scott, P. (1996). Young people's images of science. Open University Press.

Duval, R. (1995). Sémiosis et pensée humaine. P. Lang.

Gilbert, J. K. (2013). Constructing Representations to Learn in Science. In : R. Tytler, V. Prain, P. Hubber \& B. Waldrip (Eds.), Representations and Models. Sense Publishers. 193-198.

Gilly, M., Roux, J. et Trognon, A. (dir.) (1999). Apprendre dans l'interaction. Presses Universitaires de Nancy ; Publications de l'université de Provence.

Greeno, J.G. et Goldman, S.V. (1998). Thinking Practices in Mathematics and Science Learning. Lawrence Erlbaum Associates.

Grize, J.-B., (1996). Logique naturelle \& communications. PUF.

Halbwachs, F. et Torunczyk, A. (1985). On Galileo's writing on mechanics : An attempt at a semantic analysis of Viviani's scholium. Synthese, 62(3), 459-484.

Johsua, S. et Dupin, J.-J. (1993). Introduction à la didactique des sciences et des mathématiques. PUF.

Kohler, A. (2015). Elements of Natural Logic for the Study of Unnoticed Misunderstanding in a Communicative Approach to Learning. Argumentum. Journal of the Seminar of Discursive Logic, Argumentation Theory and Rhetoric, 13(2), 80-96.

Kohler, A., Chabloz, B. et Perret-Clermont, A.-N. (2015). Dispositifs d'enseignement mi-finis : une condition de collaboration entre enseignants et chercheurs?. Cahiers de psychologie et éducation (Université de Neuchâtel), 51, 5-26.

Lemeignan, G. et Weil-Barais, A. (1993). Construire des concepts en physique : l'enseignement de la mécanique. Hachette éducation.

Luc, C. et Durey, A. (1997). Modèles et modélisation dans les séquences de travaux pratiques sur le haut-parleur en classe de seconde. Didaskalia, 11, 39-73.

Martin, G. (dir.) (1988). Physique 1re S/E. Éditions Bordas. 
Martinand, J.-L. (dir.) (1992). Enseignement et apprentissage de la modélisation en sciences. Paris: INRP.

Perret-Clermont, A.-N. (1992). Les implicites dans les situations d'apprentissage. Cahiers de l'Institut Supérieur de Pédagogie de l'Université Catholique de Paris, 19, 20-53.

Perret-Clermont, A.-N. et Carugati, F. (2004). Des psychologues sociaux étudient l'apprentissage. Dans G. Chatelanat, C. Moroet, M. Saada-Robert (dir.). Unité et pluralité des sciences de l'éducation (p 159-183). Peter Lang.

Perret-Clermont, A.-N. et Schubauer-Leoni, M.-L. (1989). Social factors in learning and teaching: Towards an integrative perspective. International Journal of Educational Research, 13, 575-580.

Piaget, J. et collaborateurs. (1977). Recherches sur l'abstraction réfléchissante. Presses universitaires de France.

Pierrard, M.-A. (dir.) (2009). Enseigner les sciences expérimentales à l'école élémentaire. Physique et technologie. Éditions Bordas. Tiberghien, A. (1994). Modeling as a basis for analyzing teaching-learning situations. Learning and Instruction, 4, 71-87.

Tiberghien, A. (2003) Des connaissances naïves au savoir scientifique. Dans M. Kail et M. Fayol (dir.), Les sciences cognitives et l'école. PUF.

Tytler, R., Prain, V., Hubber, P. et Waldrip, B. (dir.) (2013). Constructing Representations to Learn in Science. Sense Publishers.

Viennot, L. (1989). Bilans de forces et loi des actions réciproques. Analyse des difficultés des élèves et enjeux didactiques. Bulletin de l'Union des Physiciens, 83, 951-971.

Vosniadou, S. (dir.), (2013). International Handbook of Research on Conceptual Change. Routledge.

Vygotski, L. (1934/1997). Pensée et langage. La Dispute.

Weil-Barais, A. (1993). L'homme cognitif. PUF.

Winther, J. (2006). Modèles et modélisation dans l'enseignement des sciences physiques. Le Bup, 100, 617-646.

Mots-clefs : Registres sémiotiques; coordination des registres sémiotiques; didactique de la physique ; situation de malentendu; modélisation

\section{Die Koordination zwischen semiotischen Registern fördern: eine Herausforderung bei Lehr-Lernprozessen in den Naturwissenschaften}

Zusammenfassung

Dieser Artikel wirft eine Frage auf, die bedeutsam für Lernprozesse im Bereich der Naturwissenschaften ist: Studierende sind mit einer Vielfalt von semiotischen Registern (Umgangssprache, Mathematik, Graphen, Schemata usw.) konfrontiert, für deren Koordination sie verantwortlich sind. Wir werden illustrieren, wie diese Aufgaben Missverständnisse hervorrufen können, indem wir mittels einer a priori Analyse Beispiele aus der Unterrichtspraxis und aus Lehrbüchern analysieren. Entsprechende Hypothesen werden anschliessend in einer Studie in Kooperation mit Lehrpersonen geprüft, indem eine unfertige Lernumgebung entworfen wird, die sich auf Modellierungskompetenzen von Schülerinnen und Schüler der Sekundarstufe II in der Schweiz konzentriert.

Aufgrund der Komplexität der Beispiele und des begrenzten Raumes für diese Publikation, präsentiert dieser Artikel nur einen exemplarischen Auszug aus den Ergebnissen. Die vorgestellte Analyse veranschaulicht die Relevanz der in den a priori Analysen identifizierten Herausforderungen für den Physikunterricht und fordert zugleich eine bewusstere und explizitere Koordination semiotischer Register bei didaktischen Entwürfen im naturwissenschaftlichen Unterricht.

Schlagworte: Semiotische Register ; Koordination semiotischer Register ; Physikdidaktik ; Situation des Missverständnisses ; Modellierung 


\title{
Coordinamento tra i registri semiotici: una questione importante per l'apprendimento delle scienze
}

\author{
Riassunto
}

Questo articolo solleva una questione e ne sostiene l'importanza nei processi di apprendimento delle scienze: gli studenti sono confrontati con una varietà di registri semiotici (linguaggio quotidiano, matematica, schemi, ecc.) che è loro responsabilità coordinare. Questo compito può indurre situazioni di incomprensione, come cercheremo di dimostrare analizzando in un primo momento alcuni esempi tratti dalle pratiche di insegnamento e dai libri di testo. L'analisi dei dati empirici relativi alle attività di apprendimento degli allievi permette, in un secondo tempo, di illustrare la rilevanza del problema individuato per l'apprendimento della fisica e invita a un uso più deliberato ed esplicito del coordinamento dei registri semiotici nella costruzione dell'ambiente didattico nell'insegnamento delle scienze.

Parole chiave: Registri semiotici; coordinamento dei registri semiotici; didattica della fisica; situazioni di incomprensione; modellizzazione

\section{Coordinating various semiotic registers: An important issue in learning science}

Summary

In this paper, we raise an issue, and support its importance within learning processes in science education : Students are confronted with a diversity of semiotic registers (daily language, mathematics, schemas, etc.) they are expected to coordinate. Such task may induce situations of misunderstanding, as we attempt to show here with a priori analyses of a few examples drawn from teaching manuals.

This idea is taken as the main hypothesis of a collaborative research, addressed to teachers through a halfbaked teaching design focusing on modeling activities for high-school students in Switzerland. This paper only presents an illustrative extract of the results, due to the complexity of the examples and the usual limit in size. The presented analysis illustrates the relevance in ordinary teaching practices, of the issue raised in the a priori analyses, and calls on a more deliberate and explicit use of the coordination of semiotic registers in the construction of a didactic environment and learning science.

Keywords: Semiotic registers; coordination; situation of misunderstanding; modelisation; Physics teaching; science education

Alaric Kohler. Après une formation en philosophie et en psychologie sociale et clinique, il écrit une thèse sur les situations de malentendu en classe de physique. Ses recherches coordonnent psychologie socio-cognitive, communication et sémiotique.

HEP-BEJUNE, chemin de la Ciblerie 45, CH-2503 Bienne

E-Mail: alaric.kohler@hep-bejune.ch

Bernard Chabloz. Physicien de formation. Après une thèse en physique théorique, il s'oriente vers l'enseignement secondaire (lycée) et la didactique (HEP-BEJUNE), avec une attention particulière pour la modélisation, l'épistémologie et le langage.

Prés de l' Envers 4, CH-2607 Cortébert

E-Mail: bchabloz@hispeed.ch 